\title{
Inklusion verlangt sozialräumlich zu denken
}

\author{
Veranstaltungsreihe der Europäischen Akademie für Inklusion
}

\author{
Diane Mönch
}

Der oft als sperrig empfundene Begriff der »Inklusion " kann auch als »Verehrung der Vielfalt « und »Aufhebung der Besonderung " übersetzt werden, stellte eine Veranstaltungsreihe der Europäischen Akademie für Inklusion fest.

In welchem Verhältnis stehen Sozialraum und Inklusion? Dieser Frage widmet die Europäische Akademie für Inklusion in Neumünster in Schleswig-Holstein eine zweijährige Veranstaltungsreihe mit dem Titel "Sozialraum und Inklusion«. Die Einrichtung war im Jahre 2008 vom Institut für berufliche Aus- und Fortbildung (IBAF gGmbH) in Rendsburg und der Elly-Heuss-Knapp-Schule gegründete worden.

Der erste Fachtag der Reihe am 31. März 2011 im Kieler Landeshaus stieß mit rund 200 Besuchern auf großes Interesse. Der Auftakt, zu dem Staatssekretärin Dr. Bettina Bonde ein Grußwort sprach, stand ganz im Zeichen der Erklärung der beiden namensgebenden Begriffe der Fachtag- und Workshopreihe, die vom Ministerium für Arbeit, Gesundheit

Diane Mönch studierte an der Universität Hamburg Soziologie und Philosophie mit den Schwerpunkten Kultursoziologie, Migrations- und Fremdenforschung sowie Religionsphilosophie und Wissenschaftstheorie mit Magisterabschluss. Während des Studiums machte sie ein Praktikum am Institut für Migrations- und Rassismusforschung in Hamburg. Bis September 2011 war sie für das Internetportal www.behinder-michnicht.de des Diakonischen Werks Schleswig-Holstein verantwortlich und konzipiert sowie organisiert für die Europäische Akademie für Inklusion die Veranstaltungsreihe "Sozialraum und Inklusion «.

Internet http://www.inklusion-sh.eu und Soziales des Landes Schleswig-Holstein im Rahmen der Initiative »Alle inklusive " gefördert wird.

Prof. Dr. Markowetz von der Katholischen Fachhochschule Freiburg grenzte »Inklusion « von »Integration « ab, die nichts anderes sei als »eine Vorstufe «, da Inklusion »weit darüber hinaus « gehe und »radikal fordere, dass Behinderung als normale Spielart menschlichen Seins in allen gesellschaftlichen Bereichen akzeptiert und entsprechend in allen administrativen Planungen regelhaft einbezogen werden muss «.

Der Vortrag zum Ansatz des Sozialraums von Peter Petersen, Referent für Soziales des Diakonischen Werks Schleswig-Holstein, orientierte sich an der »Leichten Sprache «, wodurch das komplexe wissenschaftliche Konzept, unter großer Zustimmung des Auditoriums, in eine für alle verständliche Sprache übersetzt werden konnte. Das Ziel der Europäischen Akademie für Inklusion ist es, durch den Diskurs zur »Inklusion « nicht wiederum die betroffenen Menschen auszuschließen, sondern sie aktiv an der Gestaltung der Reihe zu beteiligen, indem Praxisbeispiele vorgestellt werden und Menschen mit Behinderungen und andere vom gesellschaftlichen Ausschluss bedrohte Menschen von ihren Erfahrungen berichten.

Als Vertreterin der Initiative »Kropp für alle« schilderte Pastorin Claudia Zabel die Stolpersteine auf dem Weg, die Ortschaft Kropp in einen Sozialraum für alle zu verwandeln, da sich der Inklusionsgedanke nicht darin erschöpfe, Bürgersteige für Rollstuhlfahrer abzusenken. Gehe es darum, gemeinsam und gleichberechtigt zu leben, gebe es viele Vorbehalte gegenüber Menschen mit Behinderungen, die nun Stück für Stück durch die Initiative abgebaut werden sollen.

Um das Sozialraumkonzept an Greifbarkeit gewinnen zu lassen, stellten die »Veddeler Kiezläufer « aus Hamburg ihr Projekt vor, das von Ayhan Altun initiiert wurde, der in Hamburg im sogenannten Problem-Stadtteil Veddel lebt. Das Projekt wurde mit dem Bürgerpreis der Stadt Hamburg ausgezeichnet sowie in andere deutsche Städte als Erfolgskonzept übertragen. Die Kiezläufer sind junge Erwachsene mit Migrationshintergrund. Sie sind im Stadtteil präsent und lösen in der »Funktion des älteren Bruders « Konflikte, die durch jugendliche Drogenkonsumenten ausgelöst werden und den Bewohnern in der Vergangenheit das Gefühl gaben, im Stadtteil nicht mehr sicher zu sein. Die Kiezläufer arbeiten eng mit Ausbildungsbetrieben zusammen, um den Jugendlichen berufliche Perspektiven zu bieten und erzielen beachtliche Erfolge in der Beruhigung des Stadtteils.

Der erste Workshop am 31. Mai 2011 mit 50 Teilnehmern in Neumünster, stand unter dem Motto »Praxis trifft Inklusion « nach dem gleichnamigen Titel des Vortrages von Prof. Dr. Ingmar Steinhart. In der Veranstaltung ging es um die Frage, wie die Einrichtungen der professionellen Behindertenhilfe mit dem Konzept der Inklusion umgehen und um den damit verbundenen notwendigen Paradigmenwechsel in der professionellen Behindertenhilfe. Außerdem wurde gefragt: Was kommt nach der Auflösung von Sonderwelten? Und: Wie werden Menschen mit Behinderungen in Zukunft wohnen?

Laut Prof. Dr. Steinhart vom Institut für Sozialpsychiatrie Mecklenburg-Vorpommern und Geschäftsführer der v. Bodelschwinghsche Stiftungen Bethel bezeichnen die Wortlaute »Verehrung der Vielfalt « und »Aufhebung der Besonderung « besser das angestrebte Ziel, als der oft als sperrig wahrgenommene Begriff der Inklusion. Er beschrieb zahlreiche administrative, politische und mentale Veränderungen, die als Grundlage der Inklusion notwendig seien, und betonte, dass die Einrichtungen der professionellen Behindertenhilfe noch sehr weit von diesem Ziel entfernt seien. 
Gelebte Inklusion präsentierten die Ausbildenden des Nueva-Evaluierungsprojektes der Lebenshilfe Berlin. Es handelte sich um zwei junge Männer, die am deutschen Pilotprojekt des aus Österreich stammenden Evaluierungsprogrammes von Menschen mit Behinderungen für Menschen mit Behinderungen teilneh-
Wohnformen wie Wohngemeinschaften des betreuten Wohnens nach ihren Lebensbedingungen wie Selbstbestimmtheit und gewährter Intimsphäre befragen. Außerdem stellten die Mitglieder der Brücke Neumünster und der Brücke Schleswig-Holstein, darunter viele Menschen mit psychischen Erkrankungen so-

\section{»Inklusion kann sich nicht darin erschöpfen, die Bürgersteige für Rollstublfabrer abzusenken «}

2012 in Neumünster statt. Die Veranstaltungsreihe endet im September 2012 mit einem Fachtag in Rendsburg. Weitere Information zu Fragen der Inklusion, eine umfangreiche Linkliste, die weiteren Termine der Reihe »Sozialraum und Inklusion « sowie die Vorträge der vergangenen Veranstaltungen finden sich auf der Website der Europäischen Akademie für Inklusion: Internet http://www.inklusionsh.eu. men. "Nueva « steht für "Nutzerinnen und Nutzer evaluieren «. Im Rahmen des Ausbildungsprojektes lernen die Teilnehmer mit Lernschwierigkeiten und Behinderungen, wie sie in Interviews die Wohnheimbewohner und Bewohner anderer wie das Hamburger Projekt »Leben mit Behinderung Hamburg « ihre sozialräumlich orientierte Arbeit vor.

Der nächste Workshop mit dem Titel "Sozialraum, Alter, Jugend und die Veränderung der Stadt" findet am 6. März

\section{Die Europäische Akademie für Inklusion}

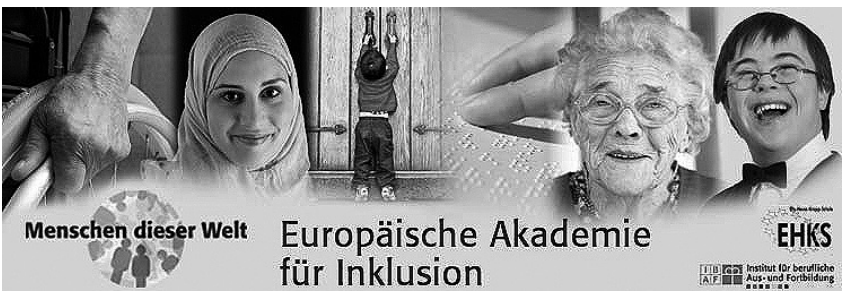

Die Europäische Akademie für Inkusion arbeitet nach dem Motto »Menschen dieser Welt «. Das Konzept der Akademie geht von der Vielfalt menschlichen Daseins aus. Die gleichberechtigte Teilhabe aller am Leben in der Gesellschaft steht dabei als Ziel im Mittelpunkt. Ethische, sozialpolitische und anthropologische Überlegungen spielen hierbei eine entscheidende Rolle. Die UN-Menschenrechtskonvention über die Rechte von Menschen mit Behinderungen aus dem Jahr 2008 und Artikel 3 des Grundgesetzes sind Beispiele für normative Beschlüsse, auf die sich die Arbeit der Akademie bezieht.

Das Konzept der Inklusion versteht die Akademie daher konsequent als alle gesellschaftlichen Bereiche betreffend. Inklusion bedeutet nicht eine Anpassung des Menschen an die Institutionen, sondern erfordert umgekehrt deren Veränderung. Diese Gedanken werden als Herausforderung und richtungweisend auf dem Weg zu einer humaneren Gesellschaft verstanden werden. Inklusion bedeutet für die Akademie die uneingeschränkte, selbstverständliche Teilhabe aller Menschen in allen Bereichen gesellschaftlichen Lebens, unabhängig von Alter, sozialem Status, Krankheit, Behinderung, ethnischer Herkunft, Geschlecht oder Religion.
Als überregionale, trägerübergreifende und parteiunabhängige Organisation hat sich die Europäische Akademie für Inklusion verschiedene Arbeitsaufgaben gestellt:

- die gesellschaftliche Auseinandersetzung mit Fragen der Inklusion kritisch begleiten

- Forschungsprojekte über Erfahrungen mit Inklusion initiieren

- praktische Erfahrungen mit Inklusion aufgreifen und reflektieren

- die Umsetzung theoretischer Ansätze in die Praxis unterstützen

- Einrichtungen vor Ort bei der Umsetzung von inklusiven Projekten unterstützen

- die interdisziplinäre Zusammenarbeit in der pädagogischen und psychosozialen Praxis befördern

- Fort- und Weiterbildungen anbieten.

Partner der Europäischen Akademie für Inklusion sind u. a. das Ministerium für Arbeit, Soziales und Gesundheit des Landes Schleswig-Holstein, der Landesbeauftragte für Menschen mit Behinderung Schleswig-Holstein, das Diakonische Werk Schleswig-Holstein, die Lebenshilfe Schleswig-Holstein, der Deutsche Paritätischer Wohlfahrtsverband Schleswig-Holstein, der Berufs- und Fachverband Heilpädagogik.

Die Europäische Akademie für Inklusion wird getragen vom Institut für berufliche Aus- und Fortbildung der Diakonie in Rendsburg und der Elly-Heuss-Knapp-Schule in Neumünster. Internet http://www.inklusion-sh.eu 